Louisiana State University

LSU Digital Commons

$5-1-2007$

\title{
Caspase activity during cell stasis: Avoidance of apoptosis in an invertebrate extremophile, Artemia franciscana
}

\author{
Michael A. Menze \\ Louisiana State University \\ Steven C. Hand \\ Louisiana State University
}

Follow this and additional works at: https://digitalcommons.Isu.edu/biosci_pubs

\section{Recommended Citation}

Menze, M., \& Hand, S. (2007). Caspase activity during cell stasis: Avoidance of apoptosis in an invertebrate extremophile, Artemia franciscana. American Journal of Physiology - Regulatory Integrative and Comparative Physiology, 292 (5) https://doi.org/10.1152/ajpregu.00659.2006 


\title{
Caspase activity during cell stasis: avoidance of apoptosis in an invertebrate extremophile, Artemia franciscana
}

\author{
Michael A. Menze and Steven C. Hand \\ Division of Cellular, Developmental and Integrative Biology, Department of \\ Biological Sciences, Louisiana State University, Baton Rouge, Louisiana \\ Submitted 18 September 2006; accepted in final form 15 January 2007
}

\begin{abstract}
Menze MA, Hand SC. Caspase activity during cell stasis: avoidance of apoptosis in an invertebrate extremophile, Artemia franciscana. Am J Physiol Regul Integr Comp Physiol 292: R2039-R2047, 2007. First published January 25, 2007; doi:10.1152/ajpregu.00659.2006.-Evaluation of apoptotic processes downstream of the mitochondrion reveals caspase-9- and low levels of caspase-3-like activities in partly purified extracts of Artemia franciscana embryos. However, in contrast to experiments with extracts of human hepatoma cells, cytochrome $c$ fails to activate caspase-3 or -9 in extracts from A. franciscana. Furthermore, caspase- 9 activity is sensitive to exogenous calcium. The addition of $5 \mathrm{mM}$ calcium leads to a $4.86 \pm 0.19$ fold (SD) $(n=$ 3 ) increase in activity, which is fully prevented with $150 \mathrm{mM} \mathrm{KCl}$. As with mammalian systems, high ATP $(>1.25 \mathrm{mM})$ suppresses caspase activity in A. franciscana extracts. A strong inhibition of caspase-9 activity was also found by GTP. Comparison of GTP-induced inhibition of caspase- 9 at 0 and $2.5 \mathrm{mM} \mathrm{MgCl} 2$ indicates that free (nonchelated) GTP is likely to be the inhibitory form. The strongest inhibition among all nucleotides tested was with ADP. Inhibition by ADP in the presence of $\mathrm{Mg}^{2+}$ is 60 -fold greater in diapause embryos than in postdiapause embryos. Because ADP does not change appreciably in concentration between the two physiological states, it is likely that this differential sensitivity to $\mathrm{Mg}^{2+}$-ADP is important in avoiding caspase activation during diapause. Finally, mixtures of nucleotides that mimic physiological concentrations in postdiapause and diapause states underscore the depressive action of these regulators on caspase- 9 during diapause. Our biochemical characterization of caspase-like activity in A. franciscana extracts reveals that multiple mechanisms are in place to reduce the probability of apoptosis under conditions of energy limitation in this embryo.
\end{abstract}

programmed cell death; apoptosome; cytochrome $c$; brine shrimp

APOPTOSIS, A FORM OF PROGRAMMED cell death, is vital for proper development and tissue homeostasis in metazoans. The regulated removal of cells enables the organism to eliminate those cells that are in excess or potentially harmful (26). In vertebrates, two major apoptotic pathways control the life or death decisions of cells. The extrinsic pathway is under control of death receptors, whereas the intrinsic pathway is triggered by mitochondria in response to intracellular injuries (18). The contribution of caspases to the apoptotic process is evolutionarily conserved $(17,41)$. Not surprisingly, activation of these cysteine proteases must be tightly regulated to avoid cell degradation under physiological conditions when it is potentially unwarranted or maladaptive. One such condition is cellular stasis, a feature of dormant stages during life cycles of many invertebrates. To gain insights into how apoptosis is avoided, we investigate here the biochemical features of

Address for reprint requests and other correspondence: M. A. Menze, Dept. of Biological Sciences, Louisiana State Univ., 202 Life Sciences Bldg., Baton Rouge, LA, 70803 (e-mail: menze@1su.edu). caspase activity in embryos of the brine shrimp Artemia franciscana, which are known to survive months in diapause without cell death $(13,20,21)$ and even longer during anoxiainduced quiescence (11).

In mammals, caspase activation in response to disturbance of intracellular homeostasis is under control of proteins that are released from the mitochondria $(33,38)$. Two mechanisms that mediate the release have been described. A delicate ratio between proapoptotic and antiapoptotic proteins of the $\mathrm{Bcl}-2$ family (B-cell lymphoma protein 2) controls the permeability of the outer mitochondrial membrane $(4,10,30)$, whereas permeability of the inner membrane is regulated by a multiprotein complex, the mitochondrial permeability transition pore (MPTP) $(3,15)$. Mitochondrial outer membrane permeabilization, due either to direct interaction with proapoptotic $\mathrm{Bcl}-2$ proteins or rupture as a result of mitochondrial swelling after MPTP opening, releases at least three factors into the cytosol that are involved in caspase activation $(25,31)$. Released cytochrome $c(\mathrm{cyt}-c)$ for example initiates the formation of the apoptosome ultimately resulting in activation of the initiator caspase, caspase-9 (casp-9), and the executioner caspase, caspase-3 (casp-3) $(5,28)$.

Embryos of the brine shrimp A. franciscana are perhaps the quintessential example of an animal resistant to severe environmental conditions, and arguments have been offered that this species should be considered an extremophile $(12,14)$. As reviewed previously (36), A. franciscana inhabits hypersaline environments such as the Great Salt Lake, Utah. In response to changes in photoperiod, population density, and/or temperature, ovoviviparous females switch to oviparous reproduction and produce encysted embryos (cysts) that enter diapause, a specific type of dormancy that is genetically programmed into life cycles and controlled by endogenous factors. Development is arrested at the gastrula stage before the embryo is released from the female's ovisac, but full metabolic depression requires an additional 4-5 days after release into the water column. These embryos are remarkable in that aerobic metabolism is depressed during diapause by as much as $97 \%$, even when oxygen, water, and temperature are sufficient to support high metabolic rates (13). The cysts remain in diapause until they are activated by the appropriate termination cues like severe dehydration or prolonged exposure to low temperature.

As shown earlier with mitochondria from A. franciscana, opening of the MPTP is refractory to calcium and all other physiological and nonphysiological chemical agents tried thus far (32). The absence of a regulated MPTP could contribute to

The costs of publication of this article were defrayed in part by the payment of page charges. The article must therefore be hereby marked "advertisement" in accordance with 18 U.S.C. Section 1734 solely to indicate this fact. 
the animal's ability to survive metabolically depressed states like diapause and environmental anoxia. During such energylimited states, the capacity to maintain mitochondrial membrane potential is impaired, intracellular calcium is expected to rise, and nucleotides such as ATP and GTP decline (7, 40). High intracellular nucleotide concentrations in mammals represent a critical pro-survival condition that prevents caspase activation $(8,9)$, whereas elevated calcium can modulate caspase activity by multiple mechanisms (40).

Thus by comparing caspase activities in cell extracts from actively developing vs. diapause embryos, we sought answers to the following specific questions: Does cyt- $c$ induce caspase activation? Although not promoting opening of the MPTP, does calcium have a more direct role in activating caspases? Is caspase activity regulated by nucleotides like ATP and GTP, which are known to decline during diapause? Answers to these questions could help identify potential safeguards that prevent triggering of apoptosis during energy-limited states in A. franciscana.

\section{MATERIALS AND METHODS}

Chemicals. Leupeptin A, pepstatin A, PMSF, DTT, ATP, ADP, GTP, GDP, horse heart cyt- $c$ and 3-[(3-cholamiddopropyl)-dimethylammonio]-1-propaneaulfonate (CHAPS) were of analytical grade from Sigma-Aldrich (St. Louis, MO). Sucrose and trehalose were purchased from Ferro Pfanstiehl Laboratories (Waukegan, IL). The caspase-9 substrate Z-LEHD-R110 and the caspase-3 substrate ZDEVD-R110 were obtained from Molecular Probes (Eugene, OR). Water for solution preparation was purified with a Milli-Q Reagent Water System (Millipore, Billerica, MA). Cell culture reagents, including PBS and trypsin solution, were obtained from Invitrogen (Grand Island, NY).

Cell culture and lysate preparation. The C3A (HepG2/C3A) human hepatoma cell line (ATCC, Manassas, VA) was grown in $75 \mathrm{~cm}^{2}$ cell culture flasks (Corning Incorporated, Corning, NY) containing standard culture medium [Eagle's minimal essential medium supplemented with $10 \%$ fetal bovine serum, 100 units $/ \mathrm{ml}$ penicillin, 100 $\mu \mathrm{g} / \mathrm{ml}$ streptomycin, $1 \mathrm{mM}$ pyruvate and $0.1 \mathrm{mM}$ nonessential amino acids (all from Invitrogen)]. Cells were maintained in a humidified atmosphere of $5 \% \mathrm{CO}_{2}-95 \%$ air at $37^{\circ} \mathrm{C}$ and allowed to reach $70-80 \%$ confluence. After one wash with PBS, cells were trypsinized for $3 \mathrm{~min}$ and resuspended in standard culture medium. The number of cells was determined by counting with a hemocytometer (Hausser and Son, Philadelphia, PA). Cells $\left(1-3 \times 10^{8}\right)$ were washed twice with ice-cold PBS and resuspended in $1.5 \mathrm{ml}$ extraction buffer (50 $\mathrm{mM} \mathrm{KCl}, 2 \mathrm{mM} \mathrm{MgCl}$, 5 mM EGTA, 10 mM DTT, 1 mM PMSF, 0.1 $\mathrm{mM}$ leupeptin, $1 \mu \mathrm{g} / \mathrm{ml}$ pepstatin A and $50 \mathrm{mM} \mathrm{K}-\mathrm{HEPES}, \mathrm{pH} 7,4)$. Cells were disrupted by using a tissue grinder (Kontes, Fisher Scientific, Houston, TX) and were centrifuged for $15 \mathrm{~min}$ at $15,000 \mathrm{~g}$ and $4{ }^{\circ} \mathrm{C}$. The supernatant was removed and again centrifuged at $100,000 \mathrm{~g}$ for $1 \mathrm{~h}$ to pellet cellular fragments. Aliquots of the supernatant were snap-frozen in liquid nitrogen and stored at $-80^{\circ} \mathrm{C}$.

Preparation of cell lysates from A. franciscana embryos. Dehydrated, encysted gastrulae of A. franciscana (originating from the Great Salt Lake, UT) were obtained from Sanders Brine Shrimp and stored at $-20^{\circ} \mathrm{C}$ until use. Embryos were hydrated in $0.25 \mathrm{M} \mathrm{NaCl}$ at $0^{\circ} \mathrm{C}$ for at least $4 \mathrm{~h}$ before incubation. Fifteen grams of hydrated embryos were then incubated at room temperature in $500 \mathrm{ml}$ of 0.25 $\mathrm{M} \mathrm{NaCl}$ on a rotary shaker at $110 \mathrm{rpm}$ for $8 \mathrm{~h}$. After this developmental incubation, the embryos were dechorionated in antiformin solution ( $1 \%$ hypochlorite from bleach, $60 \mathrm{mM} \mathrm{NaCO}_{3}$, and $0.4 \mathrm{M}$ $\mathrm{NaOH}$ ) followed by a rinse in $1 \%$ thiosulfate and multiple washings in ice-cold $0.25 \mathrm{M} \mathrm{NaCl}$, as previously described (29). After the embryos were filtered and blotted dry, $10 \mathrm{~g}$ were homogenized in 20 $\mathrm{ml}$ ice-cold isolation buffer $1(0.5 \mathrm{M}$ sucrose, $150 \mathrm{mM} \mathrm{KCl}, 5 \mathrm{mM}$ EGTA, $10 \mathrm{mM}$ DTT, $1 \mathrm{mM}$ PMSF, $0.1 \mathrm{mM}$ leupeptin, $1 \mu \mathrm{g} / \mathrm{ml}$ pepstatin $\mathrm{A}$, and $20 \mathrm{mM}$ K-HEPES, $\mathrm{pH}$ 7.5) using a glass-Teflon homogenizer at 1,000-1,100 rpm for six passages. The homogenate was centrifuged for $15 \mathrm{~min}$ at $15,000 \mathrm{~g}$ and $4^{\circ} \mathrm{C}$; the supernatant was removed and again centrifuged at $100,000 \mathrm{~g}$ for $1 \mathrm{~h}$ to pellet cellular fragments. The cytosolic fraction obtained in this way was stripped of low molecular weight compounds by using a PD-10 Sephadex G-25 column equilibrated with ice-cold buffer $2(0.5 \mathrm{M}$ trehalose and 20 $\mathrm{mM}$ HEPES-Tris, $\mathrm{pH}$ 7.2). After elution from the column, protease inhibitors ( $1 \mathrm{mM}$ PMSF, $0.1 \mathrm{mM}$ leupeptin, $1 \mu \mathrm{g} / \mathrm{ml}$ pepstatin A) and $10 \mathrm{mM}$ DTT were added to the stripped extract, and aliquots were snap-frozen in liquid nitrogen and stored at $-80^{\circ} \mathrm{C}$.

Diapause embryos were collected from the surface of the Great Salt Lake, UT, in the hydrated state, transported to the laboratory, thoroughly washed, and stored in the hydrated state, as previously described (36). Before use in experiments, the embryos were incubated 4 days at room temperature in $33 \%$ (wt/vol) Instant Ocean sea salts (Aquarium Systems, Monitor, $\mathrm{OH}$ ) on a rotary shaker at $110 \mathrm{rpm}$. This 4-day incubation allowed individuals not in diapause to hatch (36). The hatched nauplius larvae and empty shells were separated from diapause embryos using a separatory funnel and discarded. Then cell extracts for caspase assays were prepared from the remaining diapause embryos, as described above for postdiapause embryos. Protein content of lysates was quantified using a Coomassie Plus Assay Reagent (Pierce, Rockford, IL), according to the manufacturer's instructions with BSA as the standard. The typical protein concentration was around $4.8 \mathrm{mg} / \mathrm{ml}$.

Fluorescence-based measurements of caspase activity. Caspase activity was measured in 96-well plates in a fluorescence plate reader (Victor 3 Multilabel Counter, PerkinElmer, Wellesley, MA). Typically, assays of cytoplasmic extracts from A. franciscana or C3A cells (final concentration in reaction mixture, $\sim 1.5 \mathrm{mg}$ protein $/ \mathrm{ml}$ ) were carried out at $25^{\circ} \mathrm{C}$ in $500 \mathrm{mM}$ trehalose, $10 \mathrm{mM}$ DTT, $0.1 \%$ CHAPS, and $20 \mathrm{mM}$ HEPES-Tris, $\mathrm{pH} 7.2$ (buffer $A$ ). To detect caspase activity, the caspase-9 substrate Z-LEHD-R110 or the caspase-3 substrate Z-DEVD-R110 was added at a final concentration of $50 \mu \mathrm{M}$, and fluorescence was excited from above the wells. The final reaction volume was $0.1 \mathrm{ml}$. The cleavage of the rhodamine substrate was measured using an excitation filter of $485 \mathrm{~nm}$ and an emission filter of $535 \mathrm{~nm}$, and data were collected at various intervals for up to $120 \mathrm{~min}$. To test the influence of various activators and inhibitors, caspase activity in experimental samples was estimated by regression of data points in the initial linear part of the curve. Activity was then expressed as fold change relative to a control sample that contained extract and the complete reaction mixture above but lacked any activators or inhibitors. Inhibition constants were determined by fitting data with the computer program SigmaPlot 2004 for Windows version 9.01 (SPSS, Chicago, IL) to the function:

$$
f=a+\frac{b-a}{1+\left(\frac{x}{\mathrm{IC}_{50}}\right)^{n}}
$$

where $f$ is the relative activity compared with control, $x$ is the inhibitor concentration, $\mathrm{IC}_{50}$ is the inhibitor concentration at half-maximal inhibition, $a$ is the inhibited and $b$ is the uninhibited relative activity, and $n$ is the Hill coefficient. For inhibition of casp- 9 activity by $\mathrm{KCl}$, data were analyzed with a Dixon plot (Enzyme kinetics module SPSS, Chicago, IL).

Isothermal titration calorimetry. All calorimetric experiments were performed with an isothermal titration calorimeter (VP-ITC; MicroCal, Northampton, MA). Nucleotide solutions were prepared by dissolving ATP, ADP, GTP, or GDP in 0.5 M trehalose and $20 \mathrm{mM}$ HEPES, and the $\mathrm{pH}$ was adjusted with $1 \mathrm{M}$ Tris base to $\mathrm{pH}$ 7.2. GTP and GDP concentrations were checked spectrophotometrically at $\lambda=252 \mathrm{~nm}$ using an 
extinction coefficient of $\epsilon=13.7 \mathrm{mM} / \mathrm{cm}$. ATP and ADP concentrations were verified at $\lambda=259 \mathrm{~nm}$ using an extinction coefficient of $\epsilon=15.4$ $\mathrm{mM} / \mathrm{cm} . \mathrm{MgCl}_{2}$ solutions were prepared in the same buffer. Titration experiments were performed at $25^{\circ} \mathrm{C}$. The injection syringe was rotated at $310 \mathrm{rpm}$, and the time interval between injections was $400 \mathrm{~s}$. The heat change accompanying the addition of buffer to ATP and the heat of dilution of magnesium chloride were subtracted from the raw data after correction for the injection signal of buffer into buffer. Titration curves were analyzed using Origin software (MicroCal).

Calculation of total magnesium concentrations. To calculate the total magnesium concentration $[\mathrm{Mg}]_{\text {tot }}$ needed to yield a given free concentration $[\mathrm{Mg}]_{\text {free }}$ in a mixture of nucleotides, the magnesium-saturation of each nucleotide was first calculated according to: $\Theta=K \cdot[\mathrm{Mg}]_{\text {free }} /(1+$ $K \cdot[\mathrm{Mg}]_{\text {free }}$ ), where $K$ is the magnesium-binding constant for the nucleotide, as determined from the ITC experiments. The amount of total magnesium required to yield the desired $[\mathrm{Mg}]_{\text {free }}$ in a mixture of nucleotides at their respective saturation $(\Theta)$ at each nucleotide's concentration $\left[\mathrm{N}_{\mathrm{n}}\right]$ was then calculated by summing the concentrations of each nucleotide-bound magnesium $\left(\Theta \cdot\left[\mathrm{N}_{\mathrm{n}}\right]\right)$ and adding $[\mathrm{Mg}]_{\text {free: }}$

$$
[\mathrm{Mg}]_{\text {tot }}=\sum\left(\Theta \cdot\left[\mathrm{N}_{\mathrm{n}}\right]\right)+[\mathrm{Mg}]_{\text {free }}
$$

Statistical analyses. Data were analyzed by employing a one-way ANOVA and Fisher's least significant difference for pairwise comparison, $P \leq 0.05$, with Minitab 1998 (Minitab, State College, PA).

\section{RESULTS}

Caspase activity in A. franciscana is refractory to cytochrome $c$. Cell extracts from diapause embryos of A. franciscana and from C3A cells were probed for activation of DEVD-cleavage activity (casp-3-like) and LEHD-cleavage activity (casp-9-like) in response to cyt-c addition (Fig. 1, representative traces from several repetitions). Hereafter, these two activities are referred to as casp-3 and casp-9. As expected, extracts obtained from $\mathrm{C} 3 \mathrm{~A}$ cells respond with a sharp increase in casp- 3 and a less pronounced increase in casp- 9 activity after addition of $2.5 \mu \mathrm{g} / \mathrm{ml} \mathrm{cyt}-c$ in the presence of $0.5 \mathrm{mM}$ dATP and $2.5 \mathrm{mM} \mathrm{MgCl}_{2}$ (Fig. 1A). Extracts from diapause embryos of $A$. franciscana, assayed under the same conditions, were refractory to cyt-c (Fig. $1 B$ ), as were postdiapause extracts (data not shown).

Influence of $\mathrm{KCl}$ and calcium on caspase activity in A. franciscana. Caspase activation by cyt-c released from the mitochondrion can be triggered in mammals by intracellular calcium overload (3). In A. franciscana, the lack of both a calcium-induced cyt- $c$ release (32) and a cyt- $c$-induced caspase activation (this study) led us to question whether calcium might directly influence caspase activity. Because casp-9 exhibits

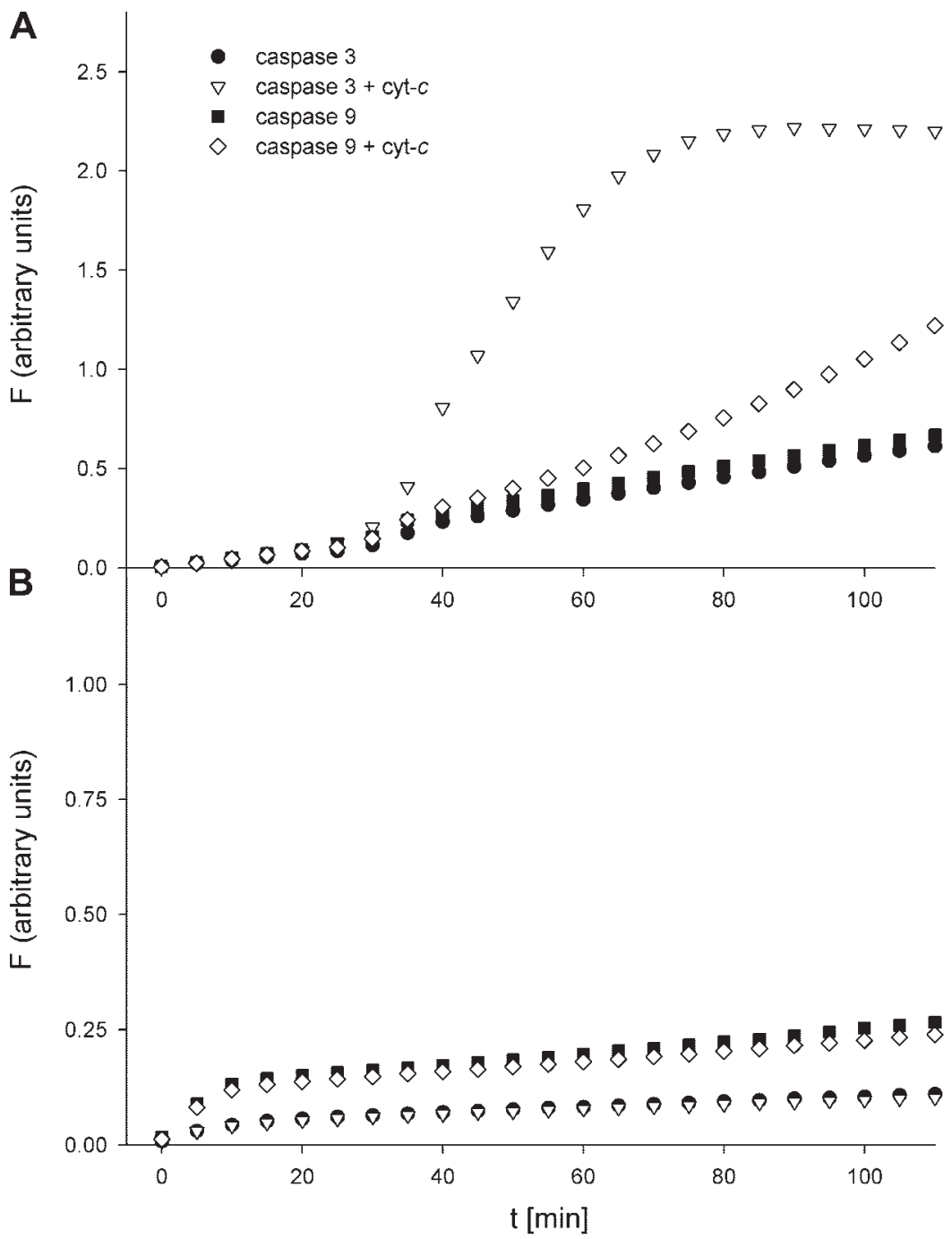

Fig. 1. Differential impact of cytochrome $c$ addition on caspase activation in cytosolic extracts from human hepatoma cells $(\mathrm{C} 3 \mathrm{~A} ; A)$ and diapause embryos of Artemia franciscana $(B)$. Caspase activities were measured at $25^{\circ} \mathrm{C}$ by following the increase in fluorescence due to cleavage of Z-LEHD-R110 (caspase 9) or Z-DEVD-R110 (caspase 3) in a 100- $\mu 1$ reaction mixture containing $500 \mathrm{mM}$ trehalose, $0.1 \%$ CHAPS, $10 \mathrm{mM}$ DTT, $15 \mathrm{mM} \mathrm{KCl}, 1.5 \mathrm{mM}$ EGTA, $2.5 \mathrm{mM} \mathrm{MgCl}, 0.5 \mathrm{mM}$ dATP, and $20 \mathrm{mM}$ HEPES-TRIS, pH 7.2. Fluorescence readings were recorded every $4 \mathrm{~min}$ for $110 \mathrm{~min}$ (representative traces of multiple measurements are shown). 
higher overall activity in A. franciscana, we focused on this enzyme for all further characterizations. Cell extracts obtained from diapause embryos show very low endogenous casp-9 activity at a $\mathrm{KCl}$ concentration of $150 \mathrm{mM}$ compared with the activity at $0 \mathrm{mM} \mathrm{KCl}$. The addition of $5 \mathrm{mM}$ calcium at this $\mathrm{KCl}$ concentration leads to no significant increase in casp-9 activity (Fig. 2, $P>0.05, n=5$ ). However, casp-9 activity in A. franciscana is inhibited by $\mathrm{KCl}$, as is caspase activation by cyt $-c$ in mammalian cells $(6,39)$. An increase in activity of $13.85 \pm 0.82(\mathrm{SD})$-fold $(n=5)$ was observed upon reducing the $\mathrm{KCl}$ concentration from $150 \mathrm{mM}$ to $0 \mathrm{mM}$ (Fig. 2). To compare activities measured under different conditions, changes were expressed with respect to the activity of a control sample measured at $0 \mathrm{mM} \mathrm{KCl}$ (Fig. 2). With decreasing $\mathrm{KCl}$ concentrations, the addition of $5 \mathrm{mM}$ calcium promotes an increased activation of casp- 9 activity. At $0 \mathrm{mM} \mathrm{KCl}$, the addition of $5 \mathrm{mM}$ calcium leads to a $4.86 \pm 0.19$ (SD)-fold $(n=3)$ increase in activity. The activation is even more dramatic (67-fold) when expressed relative to the depressed activity at $150 \mathrm{mM} \mathrm{KCl} \mathrm{(Fig.} \mathrm{2).}$

The influence of $\mathrm{KCl}$ on casp-9 activity was further investigated by measuring the activity at three different substrate concentrations $(25,50$, and $100 \mu \mathrm{M})$. Analysis with a Dixon plot shows that inhibition by $\mathrm{KCl}$ occurs with an apparent inhibitor constant of $K_{\mathrm{i}}=5.8 \pm 0.19$ (SD) $\mathrm{mM}$, and therefore casp-9 is highly inhibited at the physiological concentration of $\sim 150 \mathrm{mM}$ (23). The apparent $K_{\mathrm{m}}$ of casp-9 for LEHD-R110 was determined to be $13 \pm 3$ (SD) $\mu \mathrm{M}(n=5$; Fig. 2 inset), and all further experiments were performed at a substrate concentration of $50 \mu \mathrm{M}$.

Influence of nucleotides on casp-9 activity. Regulation of casp-9 activity in diapause $A$. franciscana was further characterized by measuring activities in the presence of increasing concentrations of ATP, ADP, and GTP (Fig. 3). Measurements were performed first in the presence of $2.5 \mathrm{mM} \mathrm{MgCl} 2$ to evaluate the influence of the naturally occurring, magnesiumchelated species. To minimize any potential interference by concurrent nucleotidase activities, casp-9 cleavage was mea- sured in 1-min intervals immediately upon the addition of extract, and rates were estimated from the linear part of the curve. The addition of $2.5 \mathrm{mM}$ magnesium in the absence of nucleotides leads to an increase in activity of $1.71 \pm 0.20$ (SD)-fold $(n=15)$. Increasing the concentrations of ATP and GTP has a biphasic influence on casp-9 activity. These two nucleotides activate at concentrations up to $0.5 \mathrm{mM}$ and inhibit at concentrations above $1.25 \mathrm{mM}$. It is known that Apaf-1mediated activation of caspases requires $\mathrm{Mg}^{2+}$-ATP for support of its ATPase activity (37). In contrast, ADP leads to a very strong inhibition of casp-9 activity at all concentrations used (Fig. 3).

The increase in casp-9 activity at low ATP and GTP concentrations depends on the availability of magnesium. Under magnesium-free conditions, diapause and postdiapause extracts are highly inhibited by $0.25 \mathrm{mM}$ ATP, GTP, or ADP, whereas GDP and AMP are far less effective at the same concentration (Fig. 4, Table 1). Cell extracts from diapause embryos do show a greater inhibition of casp- 9 activity by nucleotides compared with extracts from postdiapause embryos (Fig. 4); inhibition by ADP was $5.3 \pm 0.66$ (SD)-fold higher $(n=6)$, by ATP $3.6 \pm 1.51(\mathrm{SD})$-fold higher $(n=6)$, and by GTP $3.2 \pm 1.39$ (SD)-fold higher $(n=4)$. However, a striking difference in response to ADP in the presence of $\mathrm{Mg}^{2+}$ between diapause and nondiapause extracts was observed (Fig. 5). Analysis of the $\mathrm{IC}_{50}$ value (Eq. 1) showed that inhibition by $\mathrm{Mg}$-ADP is far greater in diapause embryos $\left[\mathrm{IC}_{50}=0.66 \pm 0.06(\mathrm{SD}) \mu \mathrm{M}, n=3\right]$ than in postdiapause embryos $\left[\mathrm{IC}_{50}=44.4 \pm 11.6(\mathrm{SD}) \mu \mathrm{M}, n=3\right]$. This observation is apt to be of physiological relevance (see DISCUSSION).

Combined influences of nucleotides on casp-9 at simulated physiological levels. The total concentration of intracellular magnesium is regulated based on the level of the free ion (27). To calculate the total magnesium concentration in a mixture of nucleotides, we determined magnesium-binding constants via ITC in the buffer employed for caspase assays (Table 2). Intracellular free $\mathrm{Mg}^{2+}$ concentrations are in the range of
Fig. 2. Caspase 9 (casp-9) activity is controlled by $\mathrm{Ca}^{2+}$ and $\mathrm{KCl}$ in cytosolic extracts from diapause embryos of $A$. franciscana. Casp- 9 activity was measured at $25^{\circ} \mathrm{C}$ in $100-\mu l$ reaction mixtures containing $500 \mathrm{mM}$ trehalose, $0.1 \%$ CHAPS, $10 \mathrm{mM}$ DTT, and $20 \mathrm{mM}$ HEPES-Tris, pH 7.2 (buffer A). Fluorescence readings were taken every minute for about 30 min, and the influence of $\mathrm{Ca}^{2+}$ and $\mathrm{KCl}$ was expressed as a fold change relative to control (see MATERIALS AND METHODS). Each value is expressed as the mean (SD) of $n=3-5$ experiments. Inset: analysis of casp-9 inhibition with a Dixon plot at 25, 50, and $100 \mu \mathrm{M}$ LEHD-R110 using 170- $\mu \mathrm{g}$ total protein indicates competitive inhibition of LEHD-cleavage by $\mathrm{KCl}(n=5)$. *Statistically different activity after calcium addition $(P<0.05)$.

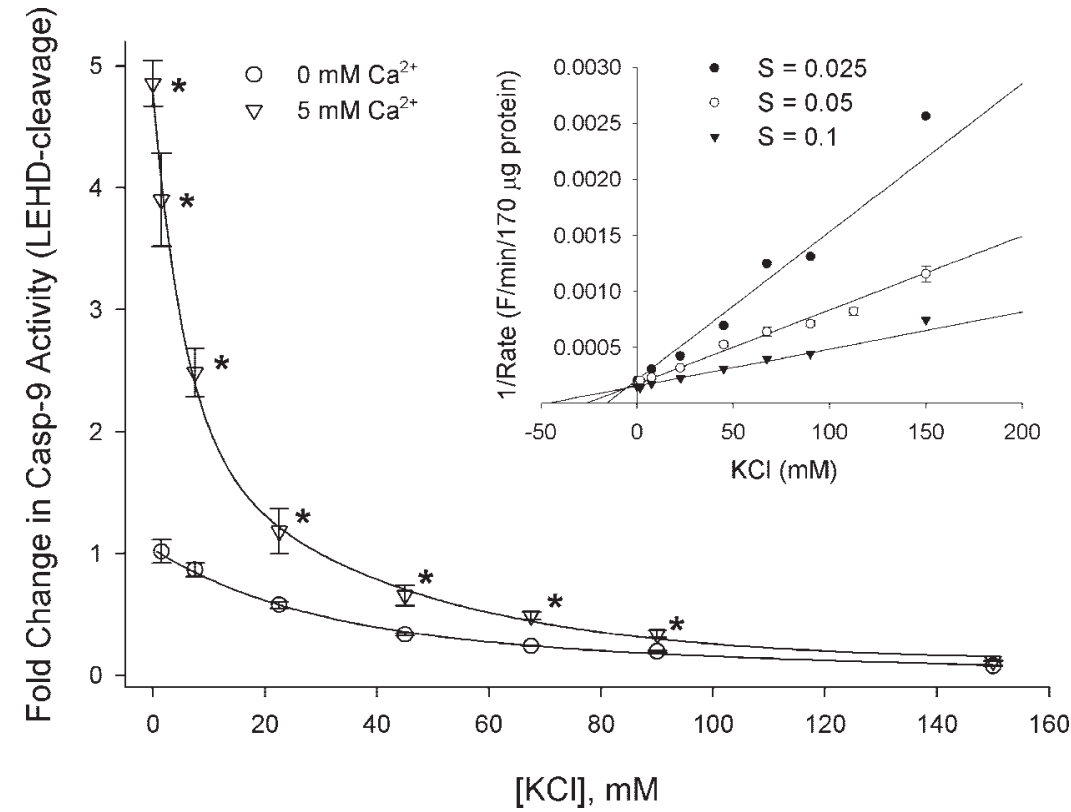

AJP-Regul Integr Comp Physiol • VOL 292 • MAY 2007 • www.ajpregu.org 


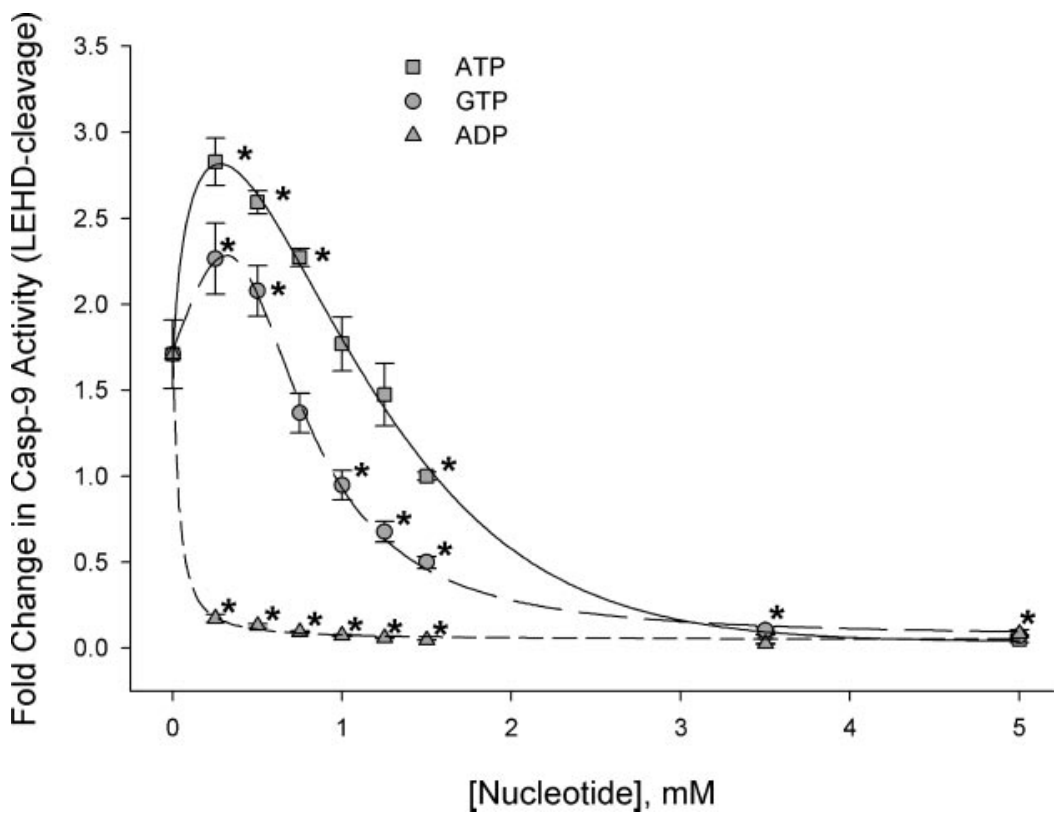

Fig. 3. Impact of ATP, GTP, and ADP on casp-9 activity (A. franciscana) in the presence of $\mathrm{MgCl}_{2}$. Addition of $2.5 \mathrm{mM}$ $\mathrm{MgCl}_{2}$ in the absence of nucleotides leads to a 1.7-fold increase in casp-9 activity in diapause extract. A biphasic pattern of activation followed by inhibition is observed for ATP and GTP. ADP is highly inhibitory at all concentrations investigated. Each value is expressed as the mean (SD) of $n=3-9$ experiments. *Statistically different to $0 \mathrm{mM}$ added nucleotides $(P<0.05)$.

$0.5-1.5 \mathrm{mM}$ for a number of cell types $(16,27)$. Typical nucleotide concentrations in postdiapause embryos are $1.2 \mathrm{mM}$ ATP, $0.26 \mathrm{mM}$ ADP, $1.4 \mathrm{mM}$ GTP, and $1.9 \mathrm{mM} \operatorname{GDP}(35,40)$. The influence on casp-9 activity of a mixture of nucleotides at the physiological concentrations estimated for postdiapause embryos was investigated at free $\mathrm{Mg}^{2+}$ concentrations of 0 $\mathrm{mM}, 0.5 \mathrm{mM}$, and $1.5 \mathrm{mM}$; AMP was not included due to the minor influence it had on caspase activity (Fig. 4). The total magnesium concentrations at these free concentrations were calculated to be $4.90 \mathrm{mM}$ and $6.13 \mathrm{mM}$ (Table. 2, Eq. 2). In the presence of the postdiapause nucleotide mixture, diapause extracts show a $\sim 60 \%$ lower casp- 9 activity compared with postdiapause extracts at both magnesium concentrations tested (Fig. 6). While nonphysiological, the inhibition by nucleotides was stronger in the absence of magnesium.

The triphosphate nucleotide and GDP concentrations in diapause embryos are substantially lower than in postdiapause embryos. Typical nucleotide concentrations in diapause embryos are $0.26 \mathrm{mM}$ ATP, $0.24 \mathrm{mM} \mathrm{ADP}, 0.42 \mathrm{mM}$ GTP, and $0.26 \mathrm{mM}$ GDP (Covi JA, Reynolds J, and Hand SC; unpublished observations). At these nucleotide concentrations, the total magnesium levels needed to yield free concentrations of $0.5 \mathrm{mM}$ and $1.5 \mathrm{mM}$ were calculated to be $1.58 \mathrm{mM}$ and 2.65 $\mathrm{mM}$, respectively (Table. 2, Eq. 2). As with the nucleotide mixture used to simulate the conditions in postdiapause embryos, caspase activity in diapause extracts is inhibited by more than $60 \%$ compared with postdiapause extracts at both magnesium concentrations used (Fig. 6). A similar depression is also estimated when caspase activity in postdiapause extracts (challenged with postdiapause nucleotide levels) is compared with the values for diapause extracts (challenged with diapause-specific nucleotide levels). This comparison is perhaps the most physiologically relevant. However, because of the reduced nucleotide levels in the diapause mixture, caspase

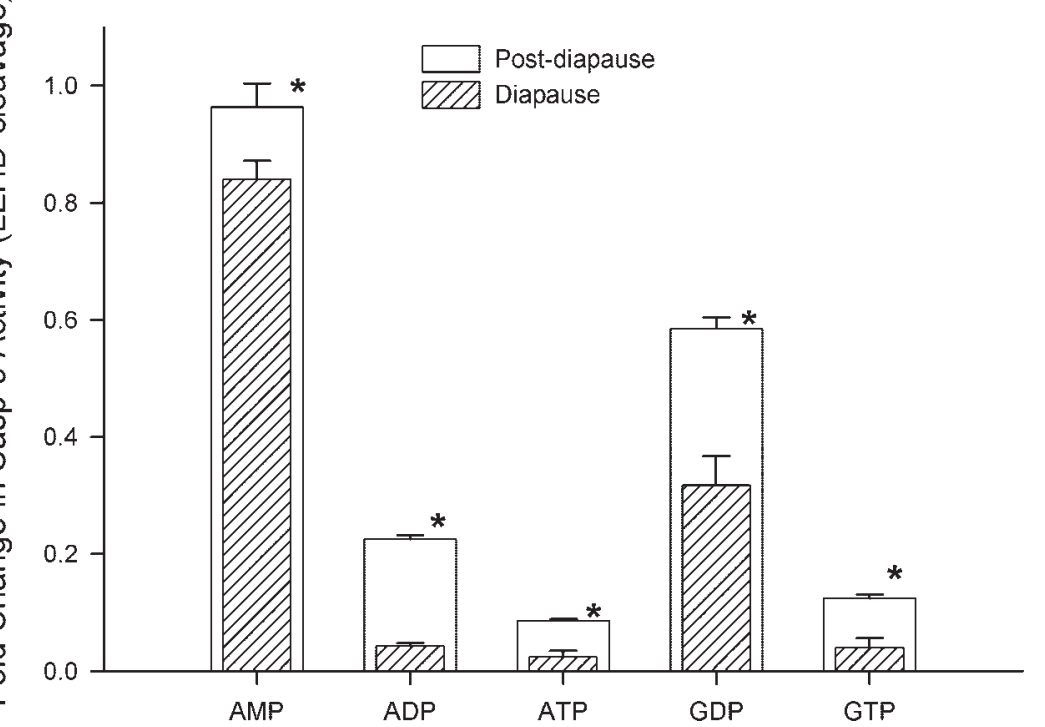

Fig. 4. Di- and tri-nucleotides inhibit casp-9 activity in the absence of $\mathrm{MgCl}_{2}$. Inhibition is higher in extracts from diapause vs. postdiapause embryos for every nucleotide investigated (buffer $A$ at $25^{\circ} \mathrm{C}$ ). Each value is expressed as the mean (SD) of $n=4-7$ experiments. *Statistically different between postdiapause and diapause $(P<0.05)$. 
Table 1. Inhibition constants for caspase-9 activity by ADP and ATP in A. franciscana cell extracts

\begin{tabular}{lcc}
\hline \hline \multicolumn{1}{c}{ Conditions } & $\mathrm{IC}_{50}, \mu \mathrm{M}$ & $R^{2}$ \\
\hline $\mathrm{ADP}, \mathrm{Mg}^{2+}$, diapause & $0.66 \pm 0.06$ & 0.997 \\
$\mathrm{ADP}$, diapause & $1.47 \pm 0.09$ & 0.999 \\
$\mathrm{ATP}, \mathrm{diapause}^{2+}$ & $0.98 \pm 0.07$ & 0.999 \\
$\mathrm{ADP}, \mathrm{Mg}^{+}$, postdiapause & $44.37 \pm 11.64$ & 0.987 \\
ADP, postdiapause & $3.11 \pm 0.35$ & 0.999 \\
ATP, postdiapause & $2.60 \pm 0.19$ & 0.999 \\
\hline
\end{tabular}

Values are expressed as means (SD) of $n=3$ experiments. Activity was followed by measuring cleavage of the fluorogenic substrate LEHD-R110, and the inhibitor constants $\left(\mathrm{IC}_{50}\right)$ were determined by nonlinear regression analysis (see MATERIALS AND METHODS). All measurements were performed with extracts (stripped of low molecular weight compounds) at $25^{\circ} \mathrm{C}$ in $0.5 \mathrm{M}$ trehalose and $20 \mathrm{mM}$ HEPES-Tris, $\mathrm{pH} 7.2$ with and without $2.5 \mathrm{mM} \mathrm{MgCl}_{2}$.

activity is always higher than when measured in a postdiapause mixture under any condition investigated (Fig. 6).

\section{DISCUSSION}

In clear contrast to mammalian systems, we have shown in this study that caspase activation by cyt- $c$ is absent in A. franciscana embryos. Second, despite this absence, many basic features of caspase regulation are observed in this crustacean. High intracellular nucleotide concentrations in mammals represent a critical prosurvival condition that prevents caspase activation due to the interaction with both cyt- $c$ (8) and casp-9 (9), whereas high intracellular potassium inhibits apoptosome formation by binding to Apaf-1 (6). Both regulatory effects are observed in A. franciscana, although the mechanisms of action may differ. Third, the regulatory influence of GTP on casp-9 is novel. Fourth, our finding that ADP blocks casp-9 more strongly in diapause vs. postdiapause extracts may represent a new mechanism to prevent caspase activation during cell stasis. In mammalian systems, a physiological role for ADP in casp-9 regulation has not been reported $(8,9)$, and ADP does not stimulate Apaf-1 activation of caspases (37). Finally, the activation of casp-9 in response to decreasing
NTP pools is less pronounced in extracts from diapause embryos compared with postdiapause embryos. Importantly, these last two phenomena could retard apoptosis during energy-limited conditions in A. franciscana.

In a previous report, we demonstrated that mitochondria from A. franciscana are resistant to calcium-induced opening of the mitochondrial permeability transition pore and subsequent cyt-c release (32). However, cyt- $c$ may also be released by mitochondrial interaction with pro-apoptotic $\mathrm{Bcl}-2$ family members (24). Therefore, we investigated whether cyt- $c$ is able to elevate casp-9 and casp-3 activities in vitro. In contrast to results obtained with $\mathrm{C} 3 \mathrm{~A}$ cell extracts, cyt- $c$ failed to elevate caspase activity in extracts from $A$. franciscana (Fig. 1, A and $B)$. Existing reports that have looked for a cyt- $c$ effect in insects appear to be contradictory on the surface. The cytosol of SF9 cells (derived from ovaries of the lepidopteran, Spodoptera frugiperda) apparently does respond to cyt- $c$ (2). However, BG2 cell extracts from D. melanogaster (derived from larval nervous system) are refractory to cyt- $c$ addition (19). The reason for the cyt- $c$ discrepancy between these insect cell lines is unclear, but perhaps the cyt- $c$ effect is restricted to germ-line cells in insects (1). A recent report on assembly and function of DARK, the apoptosome of D. melanogaster, indicates that cyt- $c$ is not required for its in vitro assembly, nor is cyt- $c$ incorporated into the final structure (42). Thus, our data are consistent with a view that the apoptosome in arthropods can assemble and activate caspases in the absence of cyt- $c$. The evolution of cyt- $c$ activation of caspases across invertebrate phyla clearly requires further investigation.

In mammals, inhibition of caspases by $\mathrm{KCl}$ is believed to occur by blocking cyt- $c$ binding to Apaf- 1 , perhaps by competing with cyt- $c$ at the WD40 site, and, in turn, prevents the processing of procaspase-9 $(2,6)$. Because A. franciscana lacks a cyt- $c$ effect, $\mathrm{KCl}$ inhibition of processing and/or recruitment of casp-9 to the apoptosome are plausible mechanisms. At low potassium concentrations, calcium increases casp-9 activity in extracts from diapause embryos, a response that is inhibited at high potassium concentrations (Fig. 2).
Fig. 5. Differential impact of $\mathrm{Mg}^{2+}$-ADP on casp- 9 activity in cytosolic extracts from diapause and postdiapause embryos of A. franciscana. In the presence of magnesium $\left(2.5 \mathrm{mM} \mathrm{MgCl}_{2}\right.$, buffer $A$ at $25^{\circ} \mathrm{C}$ ), ADP inhibition is about $70 \%$ greater in diapause extracts at the approximate physiological concentration of ADP $(15-150 \mu \mathrm{M})$ than in postdiapause extracts. Each value is expressed as the mean (SD) of $n=4-7$ experiments. * Statistically different between postdiapause and diapause $(P<0.05)$.

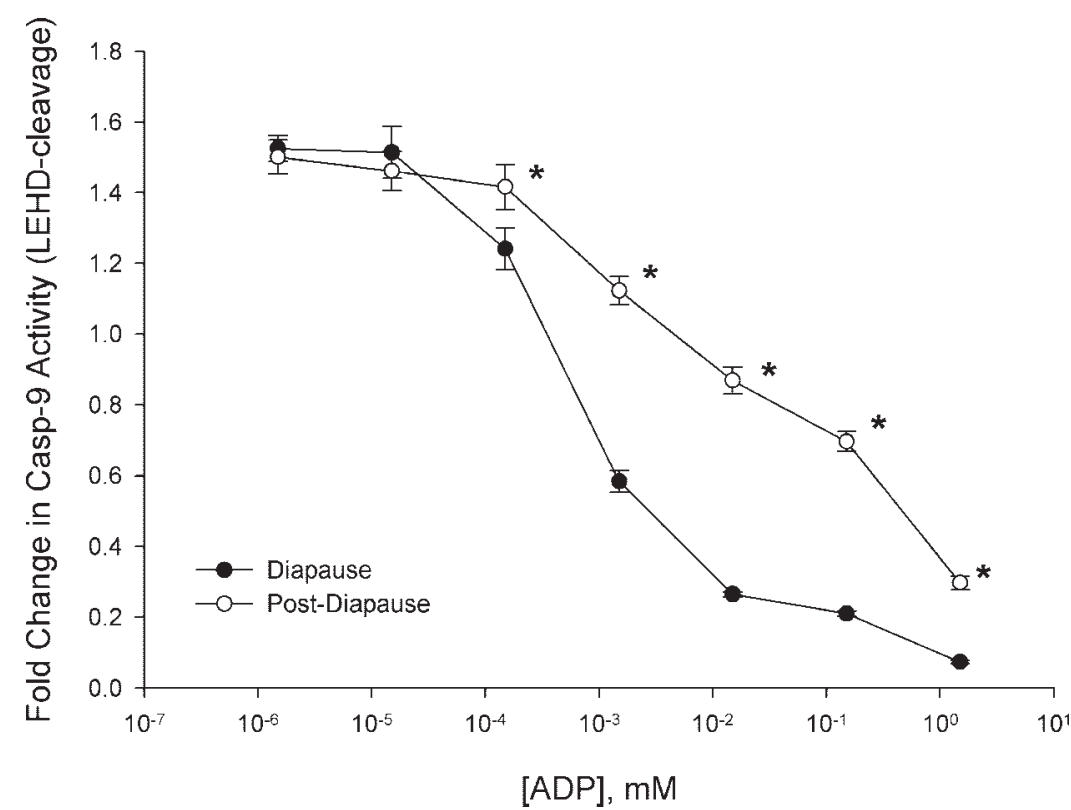

AJP-Regul Integr Comp Physiol • VOL 292 • MAY 2007• www.ajpregu.org 
Table 2. Thermodynamic parameters of magnesium binding to nucleotides as estimated by isothermal titration calorimetry

\begin{tabular}{|c|c|c|c|c|c|}
\hline Nucleotide & $n$ & $\mathrm{~K}, \mathrm{M}^{-1}$ & $\Delta \mathrm{H}^{0}, \mathrm{cal} / \mathrm{mol}^{-1}$ & $\Delta \mathrm{S}^{0}, \mathrm{cal} \cdot \mathrm{mol}^{-1} \cdot \mathrm{K}^{-1}$ & $\chi^{2}$-Test \\
\hline ADP & $1.01 \pm 0.004$ & $8,861 \pm 215.2$ & $2,569 \pm 14.92$ & 26.67 & 197.7 \\
\hline GTP & $1.00 \pm 0.006$ & $99,340 \pm 5,638$ & $1,982 \pm 17.26$ & 29.51 & 598.1 \\
\hline
\end{tabular}

The stated errors represent the uncertainties given by the fitting routine for the individual parameters. $n=$ number of binding sites. Measurements were performed at $25^{\circ} \mathrm{C}$ in $0.5 \mathrm{M}$ trehalose and $20 \mathrm{mM}$ HEPES-Tris, pH 7.2.

Calcium-activated proteases like calpain can cleave several apoptogenic proteins, most notably Apaf-1, Bax, and casp-3. This protease action leads to a decrease or increase in caspase activity (34). The calcium response of $A$. franciscana might indicate an involvement of calcium-activated proteases in the apoptotic pathway of this animal. To clarify whether the calcium response is due to proteolysis, we incubated cell extracts with $1 \mathrm{mM}$ calcium for $1 \mathrm{~h}$ at $25^{\circ} \mathrm{C}$. Calcium was then chelated by addition of EGTA. Extracts in which calcium was chelated showed only a minimal increase in activity compared with a $350 \%$ increase if calcium were not chelated (data not shown). Furthermore, activity was inhibited by more than $96 \%$ after the addition of $150 \mathrm{mM} \mathrm{KCl}$ in both cases. A proteolytic activation of casp-9 due to a calcium-activated protease is therefore unlikely, as we would expect the increase in activation to be maintained (not reversed) after calcium chelation. Thus, we speculate that the calcium effect on casp-9 is mediated allosterically.

High ATP concentrations (mM) inhibit caspase activation by direct binding to casp-9 (9). Recently, it has been reported that high concentrations of nucleotide $5^{\prime}$-triphosphates (NTPs) and 2-deoxynucleotide 5'-triphosphates (dNTPs) are effective in depressing apoptosome formation by binding to cyt- $c$ and inhibiting interaction with Apaf-1 (8). In this study, we showed that in the absence of magnesium, ATP is highly effective in inhibiting casp-9 activity (Fig. 4) with $\mathrm{IC}_{50}$ values around 1-3 $\mu \mathrm{M}$ (Table 1). In the presence of $2.5 \mathrm{mM}$ magnesium, inhibition occurs at higher ATP concentrations (Fig. 3). The free ATP concentration rises from $1.25 \mu \mathrm{M}$ at $0.25 \mathrm{mM}[\mathrm{ATP}]_{\text {tot }}$ to
$41 \mu \mathrm{M}$ at $2 \mathrm{mM}[\mathrm{ATP}]_{\text {tot. }}$. Considering an $\mathrm{IC}_{50}$ value around 3 $\mu \mathrm{M}$ for unchelated ATP, this form is likely to be the inhibitory species. Until now, very little attention has been directed toward the impact of magnesium on the regulatory features of nucleotides with respect to caspase activity.

The regulatory patterns for GTP on casp-9 in A. franciscana embryos are very similar to ATP (Figs. 3 and 4). Intracellular GTP remains high $(>2 \mathrm{mM})$ in embryos exposed to anoxia (40) and thus might serve as a means to prevent maladaptive apoptosis under prolonged hypoxia that otherwise could occur as ATP levels drop precipitously $(7,40)$. The remarkably high levels of soluble guanosines present in A. franciscana embryos are, in part, a consequence of the huge stores of $\mathrm{p}^{1}, \mathrm{p}^{4}$ diguanosine 5 '-tetraphosphate and its complex metabolic interconversions $(22,40)$. The suggestion that in mammals GTP/ dGTP may bind to cyt- $c$, prevent apoptosome formation, and thereby inhibit indirectly caspase activation (9) cannot explain the GTP effect in A. franciscana. GTP apparently can promote a more direct inhibition by binding to casp-9 or perhaps to the adaptor platform (e.g., Apaf-1/Ced-4). Surprisingly, we found that ADP in the presence of magnesium is highly effective in inhibiting casp-9 activity in extracts from diapause embryos, but much less so in extracts from postdiapause embryos (Table 1, Fig. 4). In A. franciscana embryos, ADP concentration remains constant during diapause (Covi JA, Reynolds J, and Hand SC, unpublished observations). Consequently, this pattern could prevent caspase activation under energy limitation when NTP levels fall. These individual nucleotide effects persist in mixtures of nucleotides that simulate physiological

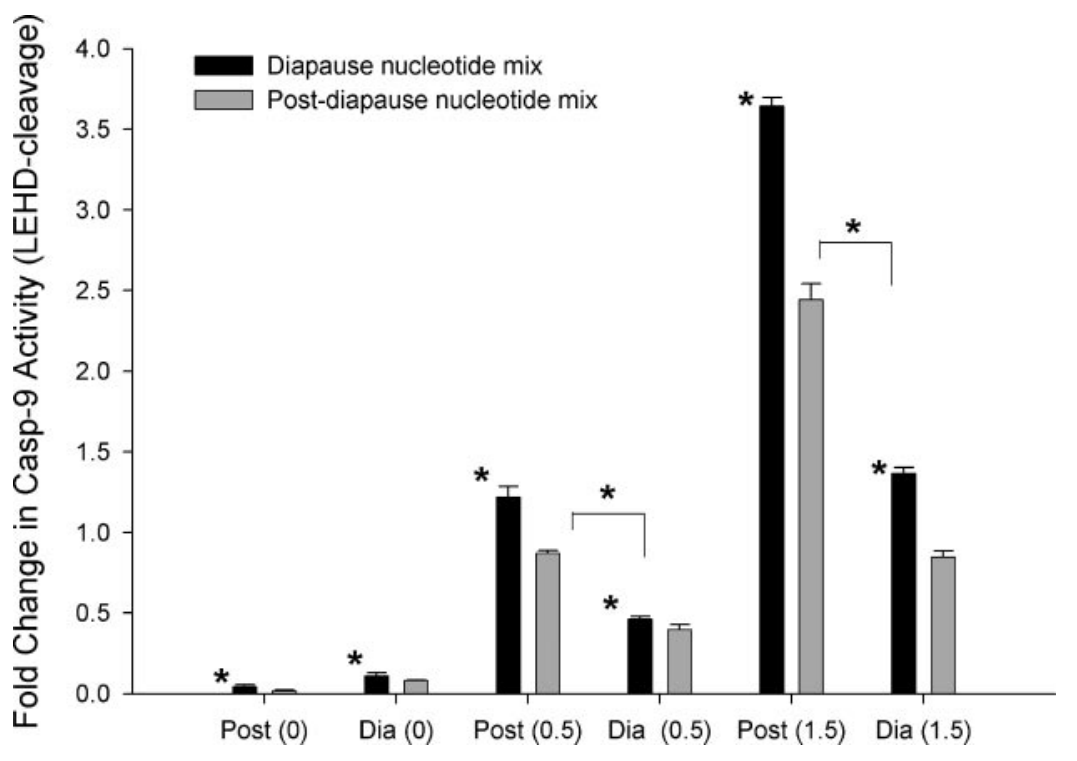

Fig. 6. Casp-9 activities in the presence of nucleotide mixtures simulating those found in postdiapause (Post) and diapause (Dia) embryos. These nucleotide mixtures were evaluated with extracts from Dia and Post embryos at $0,0.5$, and $1.5 \mathrm{mM}$ $\mathrm{MgCl}_{2}$ in buffer $A$ at $25^{\circ} \mathrm{C}$. The Post nucleotide mixture consisted of $1.2 \mathrm{mM}$ ATP, $0.26 \mathrm{mM}$ ADP, $1.4 \mathrm{mM}$ GTP, and $1.9 \mathrm{mM}$ GDP. The Dia nucleotide mixture contained $0.26 \mathrm{mM}$ ATP, $0.24 \mathrm{mM}$ ADP, $0.42 \mathrm{mM}$ GTP, and $0.26 \mathrm{mM}$ GDP. Each value is expressed as the mean $\pm \mathrm{SD}$ of $n=5-7$ experiments. *Statistically different between Post and Dia $(P<0.05)$. 
concentrations and at two different magnesium levels. Specifically, diapause extracts exhibit an overall greater depression in casp-9 activity compared with postdiapause with nucleotides mixtures that simulate either the active or the diapause state (Fig. 6).

In summary, cyt- $c$ does not promote the activation of caspases in A. franciscana embryos. Fully establishing the evolutionary patterns for the involvement of cyt- $c$ in the apoptotic cascade of eukaryotic organisms would be instructive from multiple perspectives. Integration of cyt- $c$ signaling into the carefully balanced process of caspase activation requires apparently more evolutionary steps than the acquisition of intracellular organelles like mitochondria. Relative to data for mammalian species, many basic features of caspase regulation by nucleotide and potassium concentrations are observed in A. franciscana, but new features of caspase regulation are also highlighted by this work. Our finding that unchelated GTP is able to block casp-9 activity directly is novel. Clarifying the role of magnesium chelation by nucleotides provides insight into the differential activation and inhibition of caspases by a given nucleotide like ATP. The strong ADP inhibition of caspases is noteworthy. Inhibition of casp-9 activity by GTP may help prevent maladaptive apoptosis under anoxia in A. franciscana, whereas inhibition by ADP could play a similar role of "applying the brakes" to apoptosis during diapause. The molecular basis of these effects requires further investigation. Nevertheless, understanding how apoptotic signaling is prevented in natural states of cell stasis might offer new avenues for mammalian cell stabilization and storage.

\section{GRANTS}

This study was supported by National Institute of General Medical Sciences Grant 1-RO1-GM071345-01.

\section{REFERENCES}

1. Arama E, Bader M, Srivastava M, Bergmann A, Steller H. The two Drosophila cytochrome $c$ proteins can function in both respiration and caspase activation. EMBO J 25: 232-243, 2006.

2. Beem E, Holliday LS, Segal MS. The MDa apoptosome is a critical intermediate in apoptosome maturation. Am J Physiol Cell Physiol 287: C664-C672, 2004.

3. Bernardi P, Krauskopf A, Basso E, Petronilli V, Blalchy-Dyson E, Di Lisa F, Forte MA. The mitochondrial permeability transition from in vitro artifact to disease target. FEBS Lett 273: 2077-2099, 2006.

4. Breckenridge DG, Xue D. Regulation of mitochondrial membrane permeabilization by BCL-2 family proteins and caspases. Curr Opin Cell Biol 16: 647-652, 2004.

5. Cain K, Bratton SB, Cohen GM. The Apaf-1 apoptosome: a large caspase-activating complex. Biochimie 84: 203-214, 2002.

6. Cain K, Langlais C, Sun XM, Brown DG, Cohen GM. Physiological concentrations of $\mathrm{K}^{+}$inhibit cytochrome $c$-dependent formation of the apoptosome. J Biol Chem 276: 41985-41990, 2001.

7. Carpenter JF, Hand SC. Arrestment of carbohydrate metabolism during anaerobic dormancy and aerobic acidosis in Artemia embryos-determination of pH-sensitive control points. J Comp Physiol [B] 156: 451-459, 1986.

8. Chandra D, Bratton SB, Person MD, Tian Y, Martin AG, Ayres M, Fearnhead HO, Gandhi V, Tang DG. Intracellular nucleotides act as critical prosurvival factors by binding to cytochrome $c$ and inhibiting apoptosome. Cell 125: 1333-1346, 2006.

9. Chereau D, Zou H, Spada AP, Wu JC. A nucleotide binding site in caspase-9 regulates apoptosome activation. Biochemistry 44: 4971-4976, 2005.

10. Chipuk JE, Bouchier-Hayes L, Green DR. Mitochondrial outer membrane permeabilization during apoptosis: the innocent bystander scenario. Cell Death Differ 13: 1396-1402, 2006.
11. Clegg J. Embryos of Artemia franciscana survive four years of continuous anoxia: the case for complete metabolic rate depression. J Exp Biol 200: 467-475, 1997.

12. Clegg JS. Desiccation tolerance in encysted embryos of the animal extremophile, Artemia. Integr Comp Biol 45: 714-724, 2005.

13. Clegg JS, Drinkwater LE, Sorgeloos P. The metabolic status of diapause embryos of Artemia franciscana (SFB). Physiol Zool 69: 49-66, 1996.

14. Clegg JS, Trotman CAN. Physiological and biochemical aspects of Artemia ecology. In: Artemia: Basic and Applied Biology, edited by Abatzopoulos T, Beardmore JA, Clegg JS and Soorgeloos P. Dordrecht: Kluwer Academic, 2002, p 129-170.

15. Crompton M. The mitochondrial permeability transition pore and its role in cell death. Biochem J 341: 233-249, 1999.

16. Csernoch L, Bernengo JC, Szentesi P, Jacquemond V. Measurements of intracellular $\mathrm{Mg}^{2+}$ concentration in mouse skeletal muscle fibers with the fluorescent indicator mag-indo-1. Biophys $J$ 75: 957-967, 1998.

17. Degterev A, Boyce M, Yuan J. A decade of caspases. Oncogene 22: 8543-8567, 2003.

18. Delhalle S, Duvoix A, Schnekenburger M, Morceau F, Dicato M, Diederich M. An introduction to the molecular mechanisms of apoptosis. Ann NY Acad Sci 1010: 1-8, 2003.

19. Dorstyn L, Mills K, Lazebnik Y, Kumar S. The two cytochrome c species, DC3 and DC4, are not required for caspase activation and apoptosis in Drosophila cells. J Cell Biol 167: 405-410, 2004.

20. Drinkwater LE. Regulation of embryonic diapause in Artemia: environmental and physiological signals. J Exp Zool 241: 297-207, 1987.

21. Drinkwater LE, Clegg JS. Experimental biology of cyst diapause. In: Artemia Biology, edited by Brown RA, Sorgeloos P, and Trotman CAN. Boca Raton: CRC, 1991, p 93-117.

22. Finamore FJ, Warner AH. The occurrence of P1, P4-diguanosine 5'-tetraphosphate in brine shrimp eggs. J Biol Chem 238: 344-348, 1963.

23. Glasheen JS, Hand SC. Metabolic heat dissipation and internal solute levels of Artemia-embryos during changes in cell-associated water. $J$ Exp Biol 145: 263-282, 1989.

24. Green DR, Kroemer G. The pathophysiology of mitochondrial cell death. Science 305: 626-629, 2004.

25. Green DR, Reed JC. Mitochondria and apoptosis. Science 281: 13091312, 1998.

26. Hengartner MO. The biochemistry of apoptosis. Nature 407: 770-776, 2000 .

27. Kennedy HJ. Intracellular $\mathrm{Mg}^{2+}$ regulation in voltage-clamped Helix aspersa neurones measured with mag-fura- 2 and $\mathrm{Mg}\left({ }^{2+}\right)$-sensitive microelectrodes. Exp Physiol 83: 449-460, 1998.

28. Kim HE, Du F, Fang M, Wang X. Formation of apoptosome is initiated by cytochrome $c$-induced dATP hydrolysis and subsequent nucleotide exchange on Apaf-1. Proc Natl Acad Sci USA 102: 17545-17550, 2005.

29. Kwast KE, Hand SC. Regulatory features of protein synthesis in isolated mitochondria from Artemia embryos. Am J Physiol Regul Integr Comp Physiol 265: R1238-R1246, 1993.

30. Lucken-Ardjomande S, Martinou JC. Regulation of Bcl-2 proteins and of the permeability of the outer mitochondrial membrane. $C R$ Biol 328: 616-631, 2005.

31. Martinou JC, Green DR. Breaking the mitochondrial barrier. Nat Rev Mol Cell Biol 2: 63-67, 2001.

32. Menze MA, Hutchinson K, Laborde SM, Hand SC. Mitochondrial permeability transition in the crustacean Artemia franciscana: absence of a calcium-regulated pore in the face of profound calcium storage. Am J Physiol Regul Integr Comp Physiol 289: R68-R76, 2005.

33. Newmeyer DD, Ferguson-Miller S. Mitochondria: releasing power for life and unleashing the machineries of death. Cell 112: 481-490, 2003.

34. Pelletier M, Oliver L, Meflah K, Vallette FM. Caspase-3 can be pseudo-activated by a $\mathrm{Ca}^{2+}$-dependent proteolysis at a non-canonical site. FEBS Lett 579: 2364-2368, 2005.

35. Rees BB, Ropson IJ, Hand SC. Kinetic properties of hexokinase under near-physiological conditions. Relation to metabolic arrest in Artemia embryos during anoxia. J Biol Chem 264: 15410-15417, 1989.

36. Reynolds JA, Hand SC. Differences in isolated mitochondria are insufficient to account for respiratory depression during diapause in Artemia franciscana embryos. Physiol Biochem Zool 77: 366-377, 2004.

37. Riedl SJ, Li W, Chao Y, Schwarzenbacher R, Shi Y. Structure of the apoptotic protease-activating factor 1 bound to ADP. Nature 434: 926-933, 2005.

38. Saelens X, Festjens N, Vande Walle L, van Gurp M, van Loo G, Vandenabeele $\mathbf{P}$. Toxic proteins released from mitochondria in cell death. Oncogene 23: 2861-2874, 2004. 
39. Segal MS, Beem E. Effect of $\mathrm{pH}$, ionic charge, and osmolality on cytochrome $c$-mediated caspase-3 activity. Am J Physiol Cell Physiol 281: C1196-C1204, 2001

40. Stocco DM, Beers PC, Warner AH. Effect of anoxia on nucleotide metabolism in encysted embryos of the brine shrimp. Dev Biol 27: 479-493, 1972.
41. Thornberry NA, Lazebnik Y. Caspases: enemies within. Science 281: $1312-1316,1998$

42. Yu X, Wang L, Acehan D, Wang X, Akey CW. Three-dimensional structure of a double apoptosome formed by the Drosophila Apaf-1 related killer. J Mol Biol 355: 577-589, 2006.

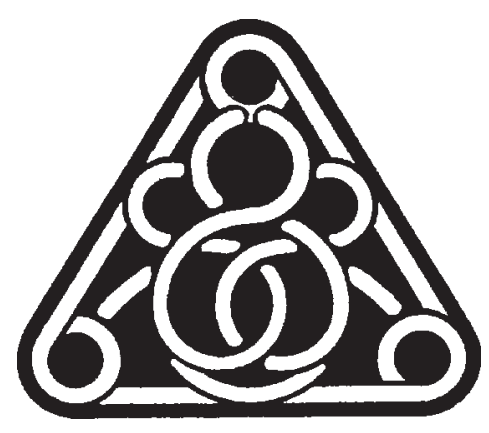

